\title{
PIE HUECO ESENCIAL DE LOS ADOLESCENTES
}

\author{
Por el Dr. ALFREDO CIFUENTES
}

Se conoce también con el nombre de idiopático, pie hrueco progresivo de los adolescentes, lo que ya nos da una idea sobre la ignorancia que hay de su etiología, al mismo tiempo que nos indica una de sus características; la de aparecer en la adolescencia o por lo menos establecerse en forma más manifiesta en esa edad. Se le denomina así mismo pie excavado, pie zambo de crecimiento o pie hueco equino, ya que en ocasiones se asocia al equinismo. Algunos autores, para indicar que en su etiología pueden intervenir muchos factores. lo rubrican con la denominación de "sindrome del pie hueco".

Esta deformación se caracteriza por una elevación del arco plantar, con dedos en garra, ligero equinismo y de aparición lenta y progresiva.

La elevación de la bóveda plantar puede efectuarse de dos maneras: acercándose ambos pilares del pie, que es lo que sucede entre los orientales por el uso de calzado más pequeño que el tamaño del pie ("pie de chino") o uno de los pilares se acerca a la bóveda del pie; si es el posterior (calcáneo). tendremos lo que se llama pie hueco posterior o paralítico, ya que su exponente más característico es el consecutivo a una parálisis del triceps. Si es el pilar anterior el que se acerca. la bóveda plantar se hace a expensas del escafoides y de las cuñas, es muy raro que se comprometa el estrágalo.

En la primera infancia, en el lactante, el pie hueco, según Max Schote, es fisiológico, pues el gateo se hace sobre la punta de los pies. Spitzy dice que, sin embargo, dan la impresión de pies planos, debido a un exceso de grasa de la planta del pie por falta de función, ya que no pisan con ella; es por esto que aconseja que anden a pie pelado o con un calzado blando.

Algunos autores consideran, para explicar esta deformación, al pie formado por 4 columnas óseas (tarso, metatarso. 
primeras y segundas falanges) equilibradas por tracciones músculo-ligamentoso, de tal modo que al fallar una de ellas, producitán deformaciones que traen consecutivamente otras por compensación.

El eje de la tibia con el antepié, forman un ángulo de unos $90^{\circ}$, el que se modífica con los movimientos flexores del pie. El antepié con el calcáneo, forma otro, que normalmente es de unos 115 a $120^{\circ}, y$, finalmente, el astrágalo con el calcáneo forman uno que oscila entre 50 y $55^{\circ}$. De las modificaciones de estos ángulos tendremos diversas variedades de pie: Pie equino, cuando aumenta el primero de ellos; pie talus. cuando sucede lo contrario: pie plano, cuando aumenta el ángulo metatarso calcáneo y si disminuye tendremos el pie hueco. el que aun puede llegar a medir menos de $90^{\circ}$. Estos ejes, vistos de atrás a adelante, se encuentran en una sola línea y de sus variaciones laterales resultaron los pies varus o valgus.

\section{Etiologia}

Nada claro ni definitivo hay respecto a la etiologia de esta afección.

La mayoría de los autores creen ver en muchos casos una espina bífida oculta, cuya única manifestación sería esta déformación, para otros, como Delchef, no sería necesaria la presencia de esta malformación, bastarian bridas, adherencias entre el raquis y las raices, estrechez del canal, es decir, lesiones extra o subdurales para producirlas, apoyando su teoria en la detención de las sustancias opacas que se observa a los Rayos $X$. Con los adelantos de la radiología cada vez se encuentra la espina bífida con más frecuencia y sin ninguna manifestación patológica, por lo que cabe preguntarse si esto no será normal o solamente un retardo en la osificación. Los que invocan como causa del pie hueco a la espina bifida, la apoyan en el becho de que cuando se interviene deliberando las raíces o descomprimiendo la médula a ese nivel, se observa una detención en la evolución del pie hueco; pero no olvidemos, dice Wallace, que hay pie buecos que carecen del factor evolutivilidad haciéndose espontáneamente estacionarios.

Otros autores, viendo que en algunas afecciones del sistema nervioso central se produce esta deformación, creeti que su causa esté en lesiones de ese sistema.

Las parálisis musculares localizadas han sido muy socorridas para explicar su aparición. Así, fara Ducroquet, sería por parálisis del flexor del dedo gordo. Royle cree que por patálisis de los gemelos el enfermo usa el tibial posterior, el 
que al flexionar el metatarso produciría el pie hueco. Duchenne cree en una parálisis de los interóseos y adductor del dedo gordo. Wallace habla de una parálisis de los lumbricales y tibial anterior. Broca hace aparecer una parálisis del flexor corto del dedo gordo. No hay duda que estas parálisis pueden producir e! pie hueco, pero no en todos los casos aparecen. Causa a qué atribuirlo no tenemos, el hecho es que se prodace un desequillibrio entre las fuerzas que actúan sobre el pie por una debilidad general del organismo sobre ta que actuarían factores externos, constituyendo lo que Mac Schotte llama insuficiencias pedias.

No hay que olvidar que esta afección tiene un cierto carácter familiar. no tiene predilección por razas ni castas, se presenta con más frecuencia en las grandes ciudades debido a la aglomeración y abundancia de centro de tratamiento.

La época de aparición es generalmente entre los 7 y 15 años. pudiendo aún hacerse presente desde los 2 años de edad o después de los 15. Es una afección, como dice Tubby, no congénita, en el sentido de nacerse con ella. A menudo es bilateral, pero siempre más acentuada en un pie, pues comienza primero en uno y a los 2 ó 3 años aparece en el otro. Que sea unilateral es may raro. En el hombre parece ser un poco más frecuente que en la mujer. Generalmente son todos ce tipo asténico.

\section{Sintomatologia}

La característica es su comienzo lento, insidioso, pero progresivo, salvo esos casos ya mencionados; estacionarios; al comienzo no bay dolores, sólo cierta inseguridad en la marcha, ésta se hace titubeante, caen con frecuencia al correr, deforman el calzado; en un período más avanzado, en 1 ó 2 años se observa una elevación en la bóveda plantar, a expensa de un acercamiento del pilar anterior, pues a la radiografía se ve que el pilar posterior (calcáneo y astrágalo) conservan sus relaciones entre sí y con la mortaja tibio peronea, es el pilar anterior el que se acerca hasta 2 y $3 \mathrm{~cm}$., la bóveda se eleva, la aponeurosis plantar, músculos y ligamentos se retraen cada vez más, el antepié se accda sobre el pie posterior a nivel del medio tarso. llegando aún a formar un ángulo recto, de tal modo que el eje de los metatarsianos llega a ser paralelo con la prolongación del eje de la pierna. La primera falange se coloca en hiperextensión, llegando su eje a ser perfendicular al de los metatarsianos: las segundas falanges se doblan, formando una $Z$ con su respectivo metatarsiano, dando el aspecto 
de dedos en garra. Todas estas deformaciones se pueden corregir en un comienzo, manualmente.

La marcha que normalmente se bace apoyando primero el talón y después el metatarso, aquí se hace al revés, primero se apoya el antepié y después el talón y en los casos avanzados ni siquiera se llega a apoyar éste en el suelo, dando la impresión de un pie equino. Como una consecuencia de esto se producen callosidades sobre la cabeza de los metatarsianos que hacen que la marcha se haga dolorosa. La huella plantar que normalmente es más marcada en el talón aqui apenas se diseñará, apareciendo la impresión del antepié más acentuada y faltando la de los dedos.

La retracción de las partes blandas de la bóveơa plantar se va acentuando hasta transformarse en una cuerda tensa, $y$ cuando el proceso es más avanzado se alteran no sólo las partes blandas sino también los ligamentos y articulaciones; se luxan las articulaciones metatarso-falángicas, se relajan los ligamentos plantares, etc.

A todo esto, en un tercio de los casos, se asocia un equinismo y un ligero varus. El estado muscular es normal, como lo demuestran las reacciones eléctricas.

Los reflejos se conservan aunque el rotuliano suele estar un poco disminuído.

\section{Diagnóstico}

El pie hueco ya descrito: progresivo con dedos en garra y ligero equinismo no ofrece dificultades diagnósticas. Podría confundirse con:

$1^{*}$ Pie hueco congénito que tiene de característica el ser muy raro, no tiene los dedos en garra, precozmente se hace jrreductible $y$, además, tenemos la radiografía para ver las deformaciones óseas.

$2^{\circ}$ Pie hueco consecutivo a cicatrices retráctiles (quemaduras, heridas, etc.) .

$3^{\circ}$ Pie bueco por flebitís y tuberculosis de la rodilla.

$4^{\circ}$ Pie hueco como síntoma de una afección dè sistema nervioso central (Little, microcefalia, diplegia cerebral infantil, enfermedad de Wilson, espasmo de torsión, pseudo esclerosis de Westphal, etc.).

$5^{\circ}$ Pie hueco consecutivo a una escaflatina, difteria o sarampión.

6. Pie hueco consecutivo a neuritis periféricas como la del saturnismo. 
$7^{\circ}$ Pie bueco como único síntoma en algunas enfer" medades.

a) Enfermedad de Friedrich, en que a veces no hav aiteraciones de los reflejos ni escoliosis, pero es fácil diferenciarlo por el carácter familiar y la bílateralidad.

b) En la enfermedad de Ledderhose hay a veces únicamente retracción de la aponeurosis plantar sin dedos en garra.

c) En la parálisis infantil que ya dijimos, que se tratzba de pie hueco posterior.

d) En las contracturas del percneo lateral largo, pero no hay dedos en garra, hay pie varus $y_{\text {t }}$ además, se puede palpar el tendón tenso.

$8^{\circ}$ Pie hueco de trinchera, for retracción de las partes blandas consecutivas a la exposición al frío de los pies.

\section{Tratamiento}

Hay diversos métodos y técnicas, pero en general todos están de acuerdo en lo que debe hacerse en un pie hueco en las diferentes etapas por las que pasa hasta hacerse inveterado 0 fijo.

El que mejor esquematiza estos tratamientos es Salvador Ciaccia, de la Clínica de Spitzy.

Para él tenemos:

1. Reducción manual.

2. Tenotomía y miotomías.

3. Operaciones sobre ét esqueleto.

4. Trasplantes tendinosos.

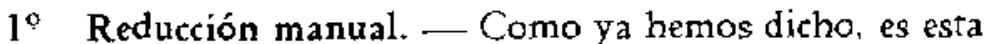
afección de lenta evolución. Si encontramos un pie hueco en su comienzo bastará recomendar el uso de un calzado alto con una buena resistencia en la garganta del pie, masajes, ejercicios de flexión y extensión del pie y el uso constante de una plantilla especial para pie hueco, $y$ con un puente anterior para levantar el antepié y con un arco plantar normal. Así, Max Schotte se inclina exclusivamente a este tratamiento, insistiendo en el uso de una plantilla de fibra elástica para que poco a poco se vaya produciendo la corrección; pues dice que la metálica corrige los síntomas persistiendo las deformaciones. Con la plantilla se modifican los puntos de apoyo del pie, desapareciendo los espasmos musculares, contracturas y callosidades que son los causantes de los dolores. Todo esto 
debe it acompañado de gran paciencia de parte del enfermo $y$ requiere el cambio frecuente de plantilla y de calzado, yā que el pie se alarga y se ensancha.

Erlacher recomienda el uso de una plantilla ideada por él y que consiste en una suela dura con un agregado para el dorso del pie unido a ésta por un material elástico para así bajat la bóveda. En los cascs rebelides o avanzados usa el tcrniquete, colocando una bota de yeso hasta la rodilla, la que secciona a nivel de la bóveda plantar $y$ aplica el torniquete desde la punta del pie a la rodilla.

Cada autor tiene su técnica para la reducción forzada usando aparatos ideados por ellos (Meyer, Still, Rolly. Codivilia, etc.).

Gocht recomienda la reducción lenta y progresiva bajo yesos sucesivos.

Schulze, Hass froponen el uso del osteoclasto.

Todos estos métodos de tratamiento dan resultados sólo en los casos de comienzo; pero tienen el inconveniente de ser poco seguros, ya que la gran mayoría se reproducen.

2" Tenotomías y miotomías. - Cuando la deformación es más avanzada y la reducción se hace irreductible se hacen necesarias otras intervenciones más radicales.

Rey Josef, Max Schotte, Stöffel recomiendan la resección de un trozo de 1 a $2 \mathrm{cms}$. del nervio plantar interno y aun del externo: que es el que comanda la retracción de los músculos que ahuecan el pie.

Como lo que más se retrae es la aponeurosis plantar se ideó su sección. En un comienzo subcutáneo, pero no es aconsejable, porque es difícil ejecutar y. además, no es suficiente. porque hay que seccionar los músculos y ligamentos más profundos, de aquí que todos recomiendan hacerla a cielo abierto seguida de una reducción forzada $e$ inmovilización larga: 2 a 4 meses.

Steinöler, Hibbs, Spitzy, etc., han ideado otra intervención que todos conocen con el nombre de "Operación de Steindler" que es la más recomendada $y$ usada. Consiste en la desinserción de la aponeurosis plantar y músculo a nivel del calcáneo a través de una incisión hecha a nivel del borde interno del pie a 1 ó $2 \mathrm{cms}$. por encima de éste o circunscribiendo el talón como aconseja Spitzy. sección de los ligamentos calcáneo-escafoídeo y calcáneo-cuboídeos. Asocian a esto una transplantación tendinosa destinada a elevar el antepié y para ello, transplantan el extensor del ptimer ortejo sobre el primer 
metatarsiano y los tendones del extensor común sobre la tercera cuña, transformándolos en flexores del pie; aun algunos transplantan el peroneo lateral largo sobre la cabeza del quinto metatarsiano o. como lo hace Bentzom, sobre el peroneo lateral corto.

Algunos autores transplantan los tendones del extensor común a los metatarsianos; pero Hibbs dice que estos tendones pierden fuerza, porque actúan paralelamente a la superficie a la que van a mover.

$3^{\text {c: }}$ Operaciones cobre el esqueleto. - Ombredanne es partidario de la operación de Steindler sólo como una intervención paliativa $y$ en niños menores de 12 años, ya que muchas veces recidivan. Alvia dice que en un tercio de los casos: Burns dice que a los 2 años se reproducen y por esto aconseja intervenciones sobre el esqueleto, que son más fieles. en sus resultados.

A pesar de esto. la mayoria son partidarios de la operación de Steindler $r_{\text {, }}$ pues sus resultados son satisfactorios para los casos de mediana gravedad y en todo caso debe hacerse como un complemento a las intervenciones sobre el esqueleto.

Para Ombredanne. cuando aun hay un pie dúctil, bastará una artrodesis media tarsiana y si éste es irreductible, hace una tarsectomía cuneiforme dorsal, que tiene el único inconveniente de acortar un poco el pie.

Wallace recomienda una osteotomía que llama tarsal anterior. evitando así la suptesión de los movimientos de lateralidad del pie ya que no compromete el astrágalo; la cuña la toma del escafoide y cuboide, por un lado. y de las cuñas por el otro. de base dorsal y de una amplitud de acuerdo con la deformación que se encuentre.

Hackembroch. Tubby, Finocchetto recomiendan una osteotomía cuneiforme dorsal sobre el primer metatarsiano para corregir la pronación del borde plantar del pie.

Hoffmann, Camera hacen osteotomía a nivel de la articulación de Lisfranc y escafoide-cuneana.

Wilson, Stuart lo hacen sólo en la de Lisfranc.

En los casos en que estas osteotomias no sean suficientes por el excesivo grado de deformación se hará necesaria una tarsectomía trancversal amplia. Para los casos más graves habrá que amputar los dedos y hacer astragalectomía. Aun describen casos en que es necesario la amputación del pie, felizmente son muy raros.

Burns y Kent Hughes recomiendan una operación ideada por Lanbrinudi, que consiste en hacer una artrodesis de las 
articulaciones interfalángicas asociadas a un trasplante cel extensor de los dedos al cuello de su metatarsiano. Para la atticulación de la primera con la segunda falange hace una artrodesis en $V$ o con un vástago y para las de $1_{a}$ segunda con la tercera sólo una resección de la superficie articular. Los dedos los mantiene en extensión, fijándolos por medio de hilos, que pasan directamente sobre la falange antes de suturar la piel para así evitar la compresión de la circulación, sobre una plantilla ideada por el. Sobre $e_{\leftarrow}^{t}$ quinto ortejo no interviene y si molesta lo amputa.

Esta intervención se asocia a la operación de Steindler como un tiempo previo y dicen que en muchos casos da tan buenos resultados que la hace innecesaria; inmoviliza por cinco semanas. No se pronuncian sobre sus resultados, pues falta el tiempo necesario para el control.

Las operaciones sobre la columna tendrán sus indicaciones precisas (espina búfida).

4" Transplante tendinoso. - Los más usados son los del extensor propio del dedo gordo sobre el primer metatarsiano, haciéndolo a través del hueso como lo recomienda Putri.

Los tendones de los extensores sea al primer metatarsiano o al tercer cuneiforme como es más aconsejable.

Sobre el tendón de Aquiles no debe intervenirse, alargándolo, sino en los casos en que esté realmente acortado para asi ni disminuir la potencia del talón.

Como un resumen, diríamos: para los casos de comienzo. masajes y plantilla. En casos más avanzados, reducción forzada en yesos por 2 a. 4 meses. Hasta los 12 años la operación de Steindler, asociada a transplantes musculares, dejando las osteotomías con todas sus variedades para los casos más avanzados.

\section{Bibliografía}

AYVIA B. - The Journal of Bone and Joint Surgery. Vol. XXIl. Fnero da 1940 .

B. H. BURNS, V. H. HELLIS. - Recenc adyances in orthopedic Surgert. London, 1937.

CAMERA (Turin). -- Técnica para e] trataminto del pie hueco. Revue DOrthopertic, 1931.

CIACCIA, SAIVADOR. - Tratamiento del pie carus. Cirugia de los ńrganes del mov.

J. DELCHEF. - Double pizd creax, resultat operatorie, Le Scalpel. Julio de 1934. 
J. HASS. - Ortopedis cruents e incruenta. Buenos Airs. 1938.

W. KENT HUGHES, - Orthopedic Surgery. Chicago. 1941.

P. J. ERLACHER. - Ortopedia operatoris. Barcelona, 1932.

PHILIF LEWTA. - The Foor ard Ankle. Filadelfia, 1940.

MASSA. - Algunos comenturios a propósito di 3 casos de pic hueco Biblio. grafía ortopédica. 1938.

T. P. MAC MURRAY A. - Practice of othopedic Surgery. Londres, 1943.

I. OMBREDANNE et MATHIEU. -- Iraité de Chirurgie orthopedique Faris. $193 \%$.

L. OMBREDANNE, - Tratado clínico de ortopedia y cirugia infantil. Batrelona, 1931.

MAC SCHOTTE. - Tuusbeschwerden Und Ihre Brhand Long. Viena, 1935 SANCHIS OLMOS. - Manual de t!inica infantil. Barcelona. 1941.

WALLACE H. COLl.ES. - Th: Journal of Bone and Joint Surgery. Voi. XXII. Octubre de $19+0$.

ROY Wh WHTMAN A. -- Treatis on orthopedie surgery. Filadefifia. 1930. 